\title{
Colonic Fistula, CTCAE
}

National Cancer Institute

\section{Source}

National Cancer Institute. Colonic Fistula, CT CAE. NCI Thesaurus. Code C143373.

A disorder characterized by an abnormal communication between the large intestine and another organ or anatomic site. 